Stefan Kühl

\section{Der Sudoku-Effekt \\ Zu den Gründen und Folgen der Komplexitätssteigerung an den Hochschulen}

\section{Zur Komplexitätssteigerung im Rahmen der Bologna-Reform}

Wenn man die Abschlusserklärung der Bildungsminister liest, die diese auf ihrer Konferenz in Bologna kurz vor der Wende zum 21. Jahrhundert verkündeten, fühlt man sich fast an einen religiösen Text erinnert. Mit großem Pathos wird unter dem Titel "Europäischer Hochschulraum « nicht weniger als ein "Europa des Wissens « versprochen, in dem Bürgern »die notwendigen Kompetenzen für die Herausforderungen des neuen Jahrtausends« vermittelt werden sollen (Bologna-Erklärung 1999: $1 \mathrm{f}$.; siehe zum religiösen Charakter z. B. Maeße 2010, 183 ff.). Auf den Folgekonferenzen der Bildungsminister wird dieses Bekenntnis in immer neuen Formulierungen wiederholt und dabei ein »inspirierendes Arbeits- und Lernumfeld" in Aussicht gestellt, das im Sinne eines "studierendenzentrierten Lernens « Studentinnen und Studenten "die besten Lösungen für nachhaltige und flexible Lernwege« bietet (BudapestWiener Erklärung 2010, 2).

Die Ziele der Studienreform à la Bologna sind dabei so formuliert, dass niemand dagegen sein kann. Studierenden soll, so das Versprechen, durch die Schaffung eines einheitlichen "Europäischen Hochschulraumes « ein höheres Maß an "Mobilität« ermöglicht werden - zwischen Fachbereichen ihrer eigenen Universität, $z$ wischen Universitäten ihres Heimatlandes und ganz besonders zwischen Universitäten in verschiedenen europäischen Staaten (BolognaErklärung 1999, 4; siehe auch Prager Erklärung 2001, 5). Dafür sollten die Studiengänge aller Universitäten gerade nicht im Sinne eines »europäischen Einheitsstudiums « homogenisiert werden, sondern es sollten lediglich alle Studienleistungen, die an einer Universität im "Europäischen Hochschulraum " erbracht werden, mit den Studienleistungen, die an anderen europäischen Universitäten eingefordert werden, kompatibel gemacht werden. Nicht Har-

1 Dieser Artikel gibt lediglich die Grundüberlegungen zum Sudoku-Effekt wieder. In dem Buch, "Der Sudoku-Effekt. Die ungewollten Nebenfolgen einer Hochschulreform «, das Anfang 2012 im transcript-Verlag erscheinen wird, werden systematisch die Folgen des Anfang $2012 \mathrm{im}$ transcript-Verlag erscheinen wird, werden systematisch die Folgen des Sudoku-Effekts aufgezeigt werden: Eine verstarkte Verschulung des Studiums, eine Verviel fältigung von (notwendigen) Regelabweichungen an den Hochschulen, sich dadurch aus bildende bürokratische Teufelskreise und eine zunehmende Diffusion von Verantwortung. Vorab sind die Druckfahnen des Buches direkt beim Autor erhältlich. monisierung sei das Ziel, so die Bildungsminister, sondern die systematische Ermöglichung eines Vergleichs aller an Universitäten erbrachten Studienleistungen durch die Bestimmung des zeitlichen Aufwandes jeder Studienleistung.

Das Mittel der Wahl zur Herstellung der Vergleichbarkeit ist - neben einem zweigliedrigen Aufbau des Hochschulstudiums in ein grundständiges Bachelorstudium und ein aufbauendes Masterstudium - besonders die verpflichtende Einführung eines Punktesystems, mit dem der Zeitaufwand der Studierenden für jede Veranstaltung, jede Prüfung, jede Laborphase, jedes Praktikum im Voraus genau kalkuliert werden soll. Dieses System mit dem etwas umständlichen Namen "European Credit Transfer and Accumulation System « - kurz ECTS - soll es ermöglichen, dass Studienleistungen, die beispielsweise an der Universität Luzern erbracht wurden, problemlos mit Studienleistungen an der Universität Bielefeld verglichen - und weitergehend dann auch gegenseitig verrechnet - werden können.

$\mathrm{Ob}$ die hehren Ziele der Bologna-Reform erreicht werden, ist heftig umstritten und wird vermutlich noch längere Zeit umstritten sein. Während Kritiker darauf verweisen, dass die angestrebte Internationalisierung der Studierenden eher gesunken als gestiegen ist (vgl. Heublein 2007, 5), wird von Befürwortern hervorgehoben, dass immer mehr Studierende die Möglichkeit nutzen, im Ausland zu studieren. Während von Kritikern mit Hinweis auf quantitative Studien darauf verwiesen wird, dass die Quote der Studienabbrecher nicht gesunken, sondern drastisch gestiegen ist (vgl. Pfaller 2010, 46), wird von Befürwortern darauf hingewiesen, dass im Rahmen der Bologna-Reform lediglich ein Studium früher abgebrochen wird und insgesamt am Ende mehr Studierende zu einem Abschluss geführt werden. Jede irgendwie verfügbare Zahl wird - andere mögliche Ursachen missachtend - auf Bologna zugerechnet, so dass sowohl die Befürworter als auch die Gegner der Bologna-Reform sich ihre nötigen Evidenzen zurechtlegen können.

Einen Effekt hat die Bologna-Reform jedoch sowohl aus Sicht der Verfechter als auch der Kritiker auf alle Fälle hervorgerufen - eine bis dahin nicht gekannte Steigerung der Komplexität. Die Komplexität der Studiengangsplanung wird inzwischen von kritischen Beobachtern mit der sozialistischen Planwirtschaft verglichen. "Wie weiland in der staatlich gesteuerten Ökonomie des Ostblocks" vergeblich versucht wurde, "die Karotten- und Stahlträgerernte der nächsten fünf Jahre bis auf die einzelne Wurzel und bis auf die konkrete Tonne Stahk vorauszuberechnen, werde jetzt, so Armin Nassehi (2009), vergeblich versucht, für alle Studiengänge einen "vollständig durchgeplanten Studienverlauf« zu erstellen.

Trotz dieser Diagnosen hatte es in den ersten Jahren der Bologna-Reform jedoch kaum Studien über die durch die Hochschulreformen ausgelöste Komplexitätssteigerung gegeben. Aber vermutlich braucht man auch keine ausgefeilte Methodik, um die Komplexitätseffekte der Bologna-Reformen zu 
bestimmen. Eine - zugegebenermaßen willkürliche - Anschauungsempirie wird dafür ausreichen.

Erstens: Bologna stellt ganz neue Anforderungen an das »Studierendenverwaltungswesen«. Die Effekte der Komplexitätssteigerung könnte man dadurch messen, dass man an den einzelnen Universitäten die Zunahme von Stellen im Bereich der Prüfungsämter, der Studiengangsverwaltung oder der Justiziariate erfasst (vgl. hierzu Winter 2009, 74). Aber häufig reicht schon der sogenannte "Schlangentest« aus. Schon beim Gang durch ein Institut oder eine Fakultät kann man mit einem Blick auf die Schlangen vor den Türen erkennen, wo die Engpässe in der Betreuung von Studierenden liegen. Die längsten Schlangen von Studierenden bildeten sich bisher vor den Türen derjenigen Professoren, die entweder besonders populär sind oder wegen ihrer Präsenz auf Fachkongressen, auf Gutachterreisen oder in den Massenmedien so selten anwesend sind, dass sie in ihren wenigen Präsenzzeiten an der Uni eine große Menge von Studierenden abfertigen müssen. Der Bologna-Prozess scheint in vielen Universitäten jetzt dazu geführt zu haben, dass sich die längsten Schlangen nicht mehr vor den Türen des Lehrpersonals bilden, sondern vor den Türen des Prüfungsamtes. Wer es nicht glaubt, möge einfach einmal selbst den Test an seiner Universität machen.

Zweitens: Studierende sind für Lehrende »Black Boxes«. Man weiß nicht genau, was in ihren Köpfen vor sich geht, womit sie sich gerade beschäftigen, was sie umtreibt. Jedoch können die Fragen, die sie an die Lehrenden richten, als grober Indikator für das dienen, was sie gerade beschäftigt. Den Komplexitätsgrad eines Studiengangs kann man deshalb daran erkennen, mit welchen Fragen Studierende zu Beginn eines Seminars, einer Vorlesung oder einer Übung auf den Lehrenden zukommen. Man kann den Eindruck bekommen, dass durch die Einführung der Bachelor- und Masterstudiengänge sich die Fragen zu einem nicht unerheblichen Teil von inhaltlichen Aspekten der Veranstaltung zu Fragen der Anrechenbarkeit verschoben haben. Nicht selten scheinen sich Lehrende ganze Sitzungen lang mit Fragen der Art zu beschäftigen, ob man in dieser Veranstaltung auch zwei Leistungspunkte mehr erwerben könne, ob statt einer Hausarbeit auch das noch durch die Studienordnung verlangte Referat gehalten werden dürfe oder an wievielen Sitzungen man teilnehmen müsse, um die aktive Teilnahme bestätigt zu bekommen.

Drittens: Den Komplexitätsgrad kann man auch daran erkennen, wie gut das Verständnis von Studiengängen ist. Die Regelungs- und Vernetzungsdichte von den sich vervielfältigenden Bologna-Studiengängen scheint inzwischen so hoch zu sein, dass Lehrende häufig selbst die eigenen Studiengänge nicht mehr verstehen. Wer sich die Dimension eines solchen Unterfangens vor Augen führen will, muss nur die im Rahmen der Bologna-Reform in Form von Studien- und Prüfungsordnungen, fächerspezifischen Bestimmungen und in Modulhandbüchern festgehaltenen diesbezüglichen Regelungen einer einzigen Universität in ihrer Papierfassung auf einen Stapel legen und diesen dann mit den Studien- und Prüfungsordnungen aus der Zeit vor Bologna vergleichen. Angesichts der Komplexität der Studiengänge können Fragen nach Leistungsnachweisen, nach Verrechenbarkeit von Modulen oder nach zu belegenden Veranstaltungen im Rahmen eines Studiengangs von den Professoren häufig selbst nicht mehr beantwortet werden. Wenn überhaupt, durchschauen nur noch die Spezialisten in der Studienberatung und in den Prüfungsämtern die Besonderheiten der jeweiligen Studiengänge. Wer dies einmal überprüfen will, muss einfach nur versuchen, sich von einem beliebigen Lehrenden den Bachelor- oder Masterstudiengang erklären zu lassen, in dem er oder sie regelmäßig unterrichtet.

Wie ist es zu dieser Komplexitätssteigerung im Zuge der Bologna-Reform gekommen? Was macht sie anscheinend so schwer beherrschbar?

Komplexität wächst nicht - wie in der frühen Komplexitätsforschung noch angenommen - alleine durch eine pure Zunahme von gleichartigen, eindeutig miteinander in Verbindung stehenden Elementen. Eine Vervielfachung von Veranstaltungen erhöht zwar die Wahlmöglichkeiten der Studierenden, verkompliziert auch die Auswahl von Veranstaltungen, macht die Studiengänge aber alleine noch nicht wesentlich komplexer. Komplexitätssteigerung entsteht vielmehr dadurch, wenn plötzlich ganz andersartige Elemente - beispielsweise neben den Veranstaltungen auch ECTS-Punkte oder Module - zusätzlich bei Entscheidungen mit in Betracht gezogen und diese Elemente auch noch auf ganz verschiedene Art und Weise miteinander in Beziehung gesetzt werden müssen (vgl. Luhmann 1980, 1064ff.).

Wir wissen aus der neueren Forschung, dass Komplexität allein durch das Zusammenspiel einiger weniger Elemente entstehen kann - und zwar dann wenn die Beziehungen zwischen den Elementen nicht genau determiniert sind (vgl. Baecker 1992). Komplexität entsteht also nicht, wenn Verknüpfung von einem Element - beispielsweise eine Vorlesung Statistik - mit genau einem anderen Element - beispielsweise einem Tutorium zur Datenerhebung - zugelassen wird. Und auch wenn alle Elemente mit allen anderen beliebig kombiniert werden können - also beispielsweise alle Veranstaltungen einer Universität kombiniert werden können - liegt bestenfalls einem »Urnebel« ähnelnde unstrukturierte Komplexität vor (vgl. Luhmann 1972, 7). Erst die Ungewissheit, mit welchen Elementen ein anderes Element verknüpft wird, schafft eine hohe strukturierte Komplexität.

Im Rahmen des Bologna-Prozesses wird eine Komplexitätsexplosion dadurch erzeugt, dass zusätzlich zu den Veranstaltungs- und Prüfungsformen mit den ECTS-Punkten und mit den Modulen neue Berechnungsformen hinzugekommen sind, die dazu führen, dass ganz neue Relationen mitbedacht werden müssen. Dabei spielt eine wichtige Rolle, dass die Module nicht jeweils nur mit einem anderen Modul kombiniert werden können, weil ein Modul in verschiedenen Studiengängen verwendet werden soll, gleichzeitig aber auch die Kombination jeden Moduls mit jedem anderen Modul - im Prinzip eine 
stark komplexitätsreduzierende Maßnahme - untersagt wird, weil Studierenden aus nachvollziehbaren Gründen nicht die beliebige Kombination so unterschiedlicher Module wie "Probleme des Genetivs und Dativs«, "Anwendungen der Integralrechnung«, »Geschichte des Nationalstaates« und »Enzymbildung« ermöglicht werden soll.

Angesichts der Notwendigkeit, die möglichen Kombinationsmöglichkeiten von den in ECTS-Punkten ausgedrückten und in Modulen zusammengefassten Veranstaltungen und Prüfungen in rechtssicheren Ordnungen der Hochschulen zu fixieren, wird die Bologna-Reform häufig als massive Bürokratisierung der Universitäten wahrgenommen. Ein »starrer Schematismus« mit »aufgeblähten Verwaltungen«, "exzessiven Modularisierungen«, »überflüssigen Akkreditierungen«, »vervielfachten Graduierungen, »unnötigen Evaluierungen«, "verwirrenden Zertifizierungen« und "zahllosen Reglementierungen« überziehe, so die Klage, „wie ein Schimmelpilz die europäischen Universitäten « (Liessmann 2009, 7). Die Zurechnung von Leistungspunkten für jeden Handgriff der Studierenden verlange von Universitäten inzwischen nicht nur eine "hochkomplexe Logistik«, sondern auch »ausgeprägte bürokratische Fähigkeiten« von Studierenden und Lehrenden (Steinert 2010, 311).

Aber wie ist es im Rahmen der Bologna-Reform zu dieser Komplexitätsexplosion in Universitäten gekommen? Weswegen hat die Zurücknahme detaillierter staatlicher Regulierungen nicht zu einer Abnahme, sondern zu einer Zunahme von Regelungen geführt?

Schnell könnte man die »üblichen Verdächtigen« für diese Regelungswut im Rahmen des Bologna-Prozesses verantwortlich machen: Als Schuldige lassen sich - wie in fast jeder Diskussion über Hochschulen - die »neoliberalen Verschwörer« heranziehen, die aus den an Humboldt orientierten Universitäten "unternehmerische Universitäten « machen wollen, in denen Bildung zu einer Ware unter anderen wird und die Studierenden zu Kunden degenerieren. Vorbereitet durch die »Lyrikwerkstatt zu Gütersloh « (Kieserling 2009, 27) und anderen nicht selten öffentlich finanzierten Zentren für Hochschulentwicklung würde, so die Beobachtung, ein »autoritär-neoliberaler Umbau der Hochschulen« auf breiter Front einsetzen (Kapfinger/Sablowski 2010, 260). Genauso wie am Markt orientierte Großunternehmen Musterfälle von bürokratischen Wucherungen geworden seien, würden dann auch die Universitäten unter dem Deckmantel einer "neoliberalen Politik« mit einem undurchschaubaren Netzwerk von Regelungen überzogen. An den Universitäten würden so lediglich Bürokratisierungsprozesse nachvollzogen, die man bei privatisierten Wasserversorgern oder bei zu Privatunternehmen umgewandelten Bahnbetreibern beobachten konnte (vgl. nur beispielhaft für den deutschsprachigen Raum und für viele andere Lieb 2009, 89 ff. oder Oelze 2010, 179 ff.).

Entgegen dieser vorschnellen Zurechnung wird in diesem Artikel argumentiert, dass die Komplexitätsexplosion an den Hochschulen, die damit verbundene Bürokratisierung des Studiums und die in den meisten Fällen damit einhergehende Verschulung nicht auf die Intentionen neoliberaler Akteure und auch nicht - wie häufig von Bildungspolitikern und Hochschulleitungen suggeriert - auf die Ungeschicklichkeit einzelner Studiengangsplaner zurückgeführt werden können. Die Komplexitätssteigerung mit einer damit einhergehenden Bürokratisierung der Studiengänge kann vielmehr - ein Konzept Robert Mertons (1936) verwendend - als "ungewollte Nebenfolge« der Einführung eines neuen Instruments der Studiengangsplanung und -steuerung identifiziert werden: Der Einführung der ECTS-Punkte als einer Art "Kunstwährung « zur Bestimmung des Arbeitsaufwandes von Studierenden. Es soll dabei nicht bestritten werden, dass die Umstellung auf ein zweigliedriges Studium, die Ersetzung von Abschlussprüfungen durch studienbegleitenden Prüfungen und die Definition von kompetenzorientierten Lernzielen - sogenannte "Learning Outcomes - jeweils eigene Probleme mit sich bringen. Aber diese Elemente der Hochschulreform haben erst in Kombination mit der Einführung der neuen Kunstwährung ECTS zu den entsprechenden Komplexitätsproblemen an den Hochschulen geführt.

\section{Zum Sudoku-Effekt - Auf der Suche nach den Gründen für die Komplexitätsexplosion}

Die Schaffung der ECTS-Punkte wird von den Promotoren als wichtiger Beitrag zur »Erhöhung der Transparenz von Lehre und Studium« gepriesen, weil sich Lehrende und Studierende »frühzeitig und zielgerichtet einen klaren Überblick« über die Studienplanung verschaffen könnten. Die Studiengänge würden insgesamt schlüssiger werden, weil durch die neue Kunstwährung die Lehrenden angehalten würden, »die Lerninhalte, die Lehrziele und die erwarteten Lehrergebnisse untereinander abzustimmen. Es komme insgesamt zu einer "Effizienzsteigerung« in den Studiengängen an den Hochschulen, weil der "Ressourceneinsat $z$ in den verschiedenen Studieneinheiten « besser kalkulierbar werde (vgl. z. B. Schwarz/Teichler 2000, 5 f.; Erhardt 2000, xi; Roscher 2000, 45).

Die Zusammenfassung von ECTS-Punkten in Modulen soll - so jedenfalls die Vorstellung der Promotoren der Bologna-Reform - Studierenden eine größere Wahlfreiheit ermöglichen (vgl. Wissenschaftsrat 2000). „Kleinere, flexibel miteinander zu verknüpfende Module« ergäben, so das Versprechen, "für die Studierenden mehr Kombinationsmöglichkeiten « als »umfangreiche Fächer« (BLK 2002, 4 und 7). Schließlich könnten die Studiengangsplaner mit der Modulstruktur nicht mehr nur disziplinäre Studiengänge für Philosophie, Wirtschaftswissenschaft und Ethnologie erarbeiten, sondern durch die $\mathrm{Zu}$ sammenstückelung von philosophischen, wirtschaftswissenschaftlichen und ethnologischen Modulen beispielsweise einen Master in sinterkultureller Wirtschaftsethik« entwickeln. Durch die Modularisierung der Studiengän- 
ge werde den Studierenden »die individuelle Gestaltung des Studiums« bei "gleichbleibender Inanspruchnahme der Kapazitäten« ermöglicht und so eine »bessere Strukturierung des Studiums« sichergestellt (KMK 2004, 1).

Es wird suggeriert, dass Studiengänge nach einem einfachen »Baukastensystem « zusammengestellt werden können, das es Studierenden ermöglicht, Module wie Legosteine miteinander zu kombinieren (vgl. zur Verwendung des Bildes des Baukastens in der Diskussion schon Weizsäcker 1970). Genauso wie Legosteine unterschiedlich viele Noppen haben können, könnten im Europäischen Hochschulraum auch die Module - je nach Arbeitsaufwand aus unterschiedlich vielen ECTS-Punkten bestehen, am Ende würden diese "Bausteine « jedoch auf eine für alle nachvollziehbare Art und Weise vielfältig kombinierbar sein. Genauso wie es dabei bei Legosteinen möglich sei, blaue, gelbe und rote Steine je nach Geschmack zusammenzusetzen, könnten zukünftig auch - wenn von den Studiengangsplanern gewünscht - in einem interdisziplinären Studiengang Module beispielsweise der Philosophie, der Wirtschaftswissenschaft, der Ethnologie, der Biologie, der Germanistik und der Sportwissenschaft miteinander kombiniert werden.

Aber das aus Modulen bestehende Baukastensystem, das durch die neue Studienstruktur produziert wird, hat, wie im Folgenden gezeigt werden soll nicht das Geringste mit diesem »Lego-Effekt« - der völlig flexiblen Kombinationsmöglichkeiten von Bausteinen - zu tun. Die Aufgabe, die in jeweils unterschiedlichen ECTS-Punkten ausgedrückten Veranstaltungsformen und Prüfungstypen in Modulen zu kombinieren, die selbst aber wiederum untereinander kombinierbar sein müssen und dabei die Gleichverteilung der zeitlichen Belastung über die Semester berücksichtigen zu müssen, ruft eher die Assoziationen mit einem Sudoku-Rätsel hervor. Abstrakt gesehen besteht ein Sudoku-Rätsel darin, dass Zahlen auf verschiedenen Ebenen - in der Horizontalen, in der Vertikalen und in den Blöcken - arithmetisch korrekt miteinander kombiniert werden müssen. Während anfangs noch ganz unterschiedliche Kombinationsmöglichkeiten vorstellbar sind, schränken sich die Kombinationsmöglichkeiten im Laufe der Füllung eines Sudoku-Rätsels immer weiter ein, so dass man am Ende froh ist, überhaupt eine Lösung gefunden zu haben.

Man darf die Sudoku-Metapher nicht übertreiben, aber die Parallelen zwischen der Gestaltung eines Sudokus und der Entwicklung eines Studiengangs sind frappierend. Genauso wie beim Sudoku innerhalb eines Blocks insgesamt Zahlen mit der Summe von $45-$ nämlich $1+2+3+4+5+6+7+8+9-$ untergebracht werden müssen, müssen auch die Vorlesungen, Seminare, Übungen, Klausuren und Hausarbeiten in Modulen mit - an vielen Universitäten vorgegebenen Größen - untergebracht werden. Genauso wie bei einer Zeile des Sudokus die Zahlen 1 bis 9 untergebracht werden müssen, muss bei der Kalkulation eines Studiums in jedem Semester die gleiche Anzahl von Leistungspunkten - in der Regel 30 ECTS-Punkte - erbracht werden.
Mit der Bildungswährung ECTS wird eine Vielzahl von Restriktionen eingeführt. Punkte können - so die Logik - letztlich immer nur erworben werden wenn in einem Modul alle jeweils mit Punkten hinterlegten Veranstaltungen und Prüfungen absolviert wurden. Weil in der Studiengangsplanung nachgewiesen werden muss, dass in der Idealform die Studierenden pro Semester nicht weniger und nicht mehr als 30 Leistungspunkte erwerben und gleichzeitig für die Seminare, Vorlesungen, Übungen und auch die Prüfungsformen möglichst immer die gleiche Anzahl von Leistungspunkten vorgesehen werden sollte, wird die Modulstruktur, die eigentlich Austauschbarkeit verspricht, unterlaufen. Die Wirkung, die dadurch - sowohl bei der Konzeption als auch bei der Lösung - produziert wird, lässt sich mit dem Mathematiker Claude E. Shannon (1951) als abnehmende Informationsentropie bezeichnen. Anfangs hat man bei der Konzeption noch alle Möglichkeiten: Man kann in ein Kästchen eine Eins, Zwei, Drei oder Acht setzen, es gibt keinerlei Restriktionen. Je mehr man jetzt bei der Konzeption voranschreitet, desto mehr Restriktionen hat man. Die Entropie nimmt immer mehr ab, bis sie am Ende gegen null geht.

Einen Studiengang muss man dabei aus drei Perspektiven betrachten: Auf der Ebene der Universitätsleitungen geht es darum, Vorgaben darüber machen, wie der Rahmen eines Studiengangs auszusehen hat. Auf der Ebene der Fakultäten, Fachbereiche oder Institute wird unter Berücksichtigung von zahlreichen formalen, aber auch inhaltlicher Vorgaben versucht, sinnvolle und akkreditierungsfähige Studiengänge zusammenzubasteln. Auf der Ebene der Studierenden versuchen diese unter Berücksichtigung der mehr oder minder schlüssigen Studienpläne, des konkreten Lehrangebots und der zeitlichen Überschneidungen von Lehrveranstaltungen ihr Studium sinnvoll zu absolvieren. Je nachdem, welche dieser drei Ebenen man betrachtet, ergeben sich dabei unterschiedliche Probleme.

Die Vorgaben, wie ein Studiengang zu modularisieren ist, welche Elemente in Modulen enthalten sein müssen und wie die Verrechnung in ECTS-Punkte stattzufinden hat, werden von den Universitätsleitungen erstellt, die sich bei ihren Vorgaben wiederum an die Richtlinien der Bildungsministerien zu halten haben. Während des Bologna-Prozesses haben sich einige Grundregeln für die Gestaltung eines Studiengangs herausgebildet. Von den Bildungsministerien der meisten Länder wird dabei vorgegeben, dass alle zeitlichen Anforderungen an Studierende in ECTS-Punkten ausgedrückt werden müssen, dass ein Studium durchgängig zu modularisieren ist und dass ein Studiengang einen in ECTS-Punkten genau festgelegten zeitlichen Umfang haben muss. Durch die Universitäten können in diesem Rahmen dann zusätzliche Vorgaben dazu gemacht werden, wie die Studiengangsentwickler ihr Studiengangs-Sudoku zu gestalten haben. Universitäten können beispielsweise Richtlinien darüber erlassen, wie die zeitlichen Anforderungen von Haupt- und Nebenfächern sich in ECTS-Punkten widerspiegeln müssen oder aus wie vielen ECTS-Punkten ein Modul mindestens bestehen muss und maximal bestehen darf. 
Im Rahmen dieser Vorgaben können die Studiengangsentwickler ein mehr oder minder kompliziertes Konzept entwickeln. Sicherlich: Bei der Konzeption eines Studiengangs kommt es selbstverständlich erst einmal darauf an, sich zu überlegen, was Studierende inhaltlich lernen sollen, mit welchen Seminaren, Vorlesungen und Übungen sie diese Lernziele am besten erreichen können und wie man das Erreichen der Lernziele am besten überprüfen kann. Durch die Einführung der Kunstwährung ECTS stehen die Studiengangsplaner aber zusätzlich vor der mathematischen Herausforderung, die Veranstaltungen, Prüfungen und Praktika mit Zahlen zu hinterlegen und so miteinander zu verknüpfen, dass am Ende für den gesamten Studiengang, aber auch für die einzelnen Semester und - an einzelnen Universitäten - auch für die einzelnen Module genau die vorgegebene Summe ECTS-Punkte herauskommt. Dafür verändern sie die Anzahl von Modulen, die Zuweisung von Prüfungen zu den Modulen, die Bewertung von Modulen, Veranstaltungen und Prüfungen mit Leistungspunkten so lange, bis am Ende je nach Vorgabe genau 240, 180, 120 oder 60 Leistungspunkte erreicht werden. Wenn sie den Studierenden Wahlmöglichkeiten lassen wollen und sich bei der Konzeption des Studiengangs geschickt anstellen, lassen sie mehrere Lösungen ihres Studiengangs-Sudokus zu, so dass die Studierenden - vielleicht sogar noch mit Hilfestellungen der Konzepteure - mehrere Lösungsstrategien einschlagen können.

Auch beim Belegen eines Studiengangs mag für Studierende erst einmal im Vordergrund stehen, Wege zu finden, ihre fachliche Neugierde zu befriedigen, sich durch neue Fragen irritieren zu lassen und eigene Wege zur Beantwortung dieser Fragen zu finden. Aber diese Interessen müssen sie notgedrungen mit den Anforderungen der ECTS-Zahlenarithmetik der Studiengänge in Einklang bringen. Bei der Lösung ihres Studiengangs-Sudokus stehen die Studierenden vor der Herausforderung, die Module, Veranstaltungen und Prüfungen so miteinander zu verknüpfen, dass sie am Ende die geforderte Anzahl an ECTS. Punkten gesammelt haben. Die Studierenden müssen die immer kleinteiligeren Anforderungen des Studiums bewältigen und dabei die formalen Restriktionen des Studienplans mit dem konkreten, in Umfang und Attraktivität wechselnden Veranstaltungsangebot von Semester zu Semester abgleichen, sowie mit weiteren zeitlichen Restriktionen wie Überschneidungen zwischen Veranstaltungen, Praktika, Nebenjobs. Da manche Veranstaltungen für mehrere Module geöffnet sind, können sich Studierende durch geschicktes "Herumschieben « und laufende Reorganisation der Zuordnung von belegten Veranstaltungen zu Modulen ein bisschen Flexibilität bewahren. Dabei kann es ihnen - wie bei einem Sudoku - passieren, dass sie zu Beginn durch das unbernerkte »Fehlbelegen« von Veranstaltungen und Prüfungen auf eine falsche Spur geraten. Das stellen sie dann häufig erst am Ende fest, wenn ihr Studium jedenfalls "punktemäßig« nicht aufgeht. Mühsam müssen dann Veranstaltungen neu Modulen zugerechnet, neue Verrechnungen von Prüfungen ausprobiert oder sogar Veranstaltungen oder Prüfungen nachgeholt werden.
Um Probleme bei der Lösung eines Studiengangs-Sudokus zu verhindern kann man es Studierenden einfach machen - und zwar dadurch, dass man ihnen den Lösungsweg direkt vorgibt. Man überlegt sich für einen einzigen Studiengang einer einzelnen Universität, welche Module mit welchen Vorlesungen, Übungen und Seminaren die Studierenden sinnvollerweise studieren sollen, wie diese Module am besten abgeprüft werden können und kalkuliert, wie viel Leistungspunkte für jeden Veranstaltungstypen, jede Prüfungsform und jedes Modul notwendig sind. Man muss lediglich darauf achten, dass man bei der Addition am Ende bei einem Bachelorstudiengang auf 180 Leistungspunkte und bei einem Masterstudiengang auf 120 Leistungspunkte kommt. Den Studierenden gibt man dann genau vor, wie sie was in welchem Semester zu studieren haben.

Dies ist der Grund, weswegen der Sudoku-Effekt bei schon immer stark verschulten Studiengängen wie Jura, Medizin, Betriebswirtschaftslehre oder den Ingenieurswissenschaften deutlich weniger häufig zu beobachten ist als beispielsweise in den Sozial- und Geisteswissenschaften. Die erstgenannten Studiengänge waren schon in ihrer Fassung als Diplom- oder Staatsexamensstudiengänge häufig so stark reguliert und verfügen über so wenig Kontaktstellen zu anderen Studiengängen, dass die Umrechnung jeder Arbeitsstunde in ECTS-Punkte nicht die gleichen Effekte zeigte wie bei den Geistes- und Sozialwissenschaften. Es gibt zwar bei der Konzeption eines Studiengangs nach wie vor einen Sudoku-Effekt, weil am Ende ja nach einem bestimmten Schema 120, 180 oder 240 Leistungspunkte herauskommen müssen, aber der Effekt wird von den Studierenden gar nicht bemerkt, weil den Studierenden ja die Lösung Schritt für Schritt vorgegeben wird, indem ihnen - wie früher auch schon - im Detail vorgeschrieben wird, wo sie an welchem Tag zu welcher Uhrzeit welche Veranstaltung zu belegen haben. Studiengänge ähneln dann einem Sudoku, bei dem den Rätsellösenden von oben Zeile für Zeile diktiert wird, mit welcher Zahl sie das jeweils nächste leere Kästchen zu füllen haben.

Sobald Studiengangsplaner jedoch die Bologna-Rhetorik - Flexibilisierung der Studiumsgestaltung, Erhöhung der Mobilität und Schaffung von Wahlfreiheiten für Studierende - ernst nehmen und dieses Ziel in die Formalstruktur von Studiengängen umzusetzen versuchen, wird es richtig kompliziert. Gerade bei der Verkopplung mehrerer Studiengänge sind die kognitiven Leistungsgrenzen der Studiengangsplaner schnell erreicht. Als Fluchtpunkt bleibt häufig nur, dass eine sich in Leistungspunkten verlierende Lehrplan-Arithmetik die Debatten über die Ausrichtung von Studiengängen überlagert.

Wenn man bei der Konzeption eines Studiengangs nur ausreichend verschiebt, modifiziert und neu berechnet, dann geht es am Ende irgendwie auf. Bloß: Genauso wie beim Sudokurätsel die Anordnung der Zahlen zwischen 1 und 9 letztlich nur durch die notwendige Vernetzung mit anderen Zahlenreihen begründet ist, wird auch bei der Gestaltung eines Studiengangs die 
Anordnung von Modulen, Veranstaltungen und Prüfungen häufig am Ende nur noch von den Konsistenzanforderungen der Leistungspunktelogik getragen. Die durch die Bologna-Reform entstandenen Studiengänge sind dann häufig nicht das Ergebnis eines Diskussionsprozesses darüber, was Absolventen eines Studiums beherrschen sollen, sondern eher das Ergebnis der permanenten Anpassung der ursprünglich einmal angedachten Veranstaltungen an die vorgegebenen starren Berechnungsschemata. Die Haltung von Studiengangsentwicklern - und dann schließlich auch von den Studierenden selbst - ist: Hauptsache, das Studium geht zahlenmäßig irgendwie auf.

\section{Verschulung als ungewollten Nebenfolgen des Sudoku-Effekts}

Die Komplexitätsexplosion durch den Sudoku-Effekt kann an den Hochschulen vermutlich durch eine weitere Vermehrung von Verwaltungs- und Planungsstellen in der Lehre und durch eine Verlagerung der Aufmerksamkeit der Lehrenden von inhaltlichen Fragen auf die Arithmetik von Studiengängen aufgefangen werden. Interessanter ist jedoch, dass der durch die Einführung von Leistungspunkten produzierte Sudoku-Effekt eine Verschulung als weitere ungewollte Nebenfolge mit sich bringt.

Mit dem Etikett der »Verschulung « wird dabei eine Vielzahl von Phänomenen erfasst: »Fixe Stundenpläne «, »klassenorientierte Lehr- und Lernorganisation«, "Anleitung statt selbstorganisiertes Lernen", "permanente Anwesenheitspflichten einhergehend mit einer hohen Kontrolldichte", "Prüfungsinflation«, "wenig Wahlfreiheiten " und "Vermittlung von kanonisiertem ,Schuk-Wissen" (Winter 2009, 49). Um zu begreifen, wie sich Verschulungstendenzen an den Hochschulen - häufig entgegen der ursprünglichen Intention der Studiengangsplaner - ausbilden konnten, erscheint es lohnend, sich beispielhaft die Zunahme von Vorlesungen, die Inflation von Prüfungen und die Einschränkung von Wahlmöglichkeiten genauer anzusehen.

Die wachsende Zahl von Vorlesungen, in denen den Studierenden Wissen nur zur passiven Aneignung angeboten wird, auf Kosten von Seminaren, in denen Inhalte selbst erarbeitet und kritisch diskutiert werden, wird als zentrales Merkmal für eine Verschulung gesehen. Die Vermehrung der Vorlesungen wird dabei häufig nicht mit didaktischen oder methodischen Überlegungen begründet (siehe dazu Stichweh 1994, 340), sondern ergibt sich aus einer von ECTS-Punkten ausgehenden Studiengangsplanung. Die Studiengangsplaner überlegen, wie viele Veranstaltungen sie in den zur Verfügung stehenden ECTS-Punkten unterbringen können und erstellen daraus ein erstes Lehrtableau. Dann sehen sie sich natürlich vor die Herausforderung gestellt, diese Veranstaltungen auch mindestens jährlich anbieten zu müssen. Weil die Veranstaltungen - ganz »studierendenzentriert - aber nicht von den zur Verfügung stehenden Lehrkapazitäten, sondern von den für die Studierenden als sinnvoll erachteten Inhalten aus geplant wurden, müssen plötzlich mehr Veranstaltungen angeboten werden als durch die Lehrkapazitäten abgedeckt werden können

Selbst sehr gut ausgestattete Institute, Fakultäten und Fachbereiche, die es an einigen europäischen Universitäten immer noch gibt und die vor den im Namen von Bologna vorgenommenen Reformen immer mit ihren Lehrkapazitäten ausgekommen sind, sollen es bei der Umstellung auf Bachelor- und Masterstudiengänge geschafft haben, dass ihnen pro Semester die Lehrkapazitäten für 20 oder 30 Veranstaltungen pro Studiengang fehlten. Man hatte jetzt zwar Studiengänge, die fast nach einem hochschuldidaktischen Idealtypus von allgemeinen Lernzielen auf Module und dann weiter auf Veranstaltungen heruntergebrochen waren, aber es fehlte einfach an Kapazitäten, mit denen die Veranstaltungen bedient werden konnten. Es bleibt dann häufig nur noch die Möglichkeit, immer mehr Studierende in die Veranstaltungen hineinzuquetschen. Weil Übungen oder Seminare ab einer Teilnehmerzahl von 50 oder 60 Studierenden keinen Sinn mehr machen, werden diese dann aus der Not heraus in Vorlesungen umdefiniert.

Ähnliche Effekte sind bei der Vermehrung von Prüfungsleistungen zu beobachten, die die Studierenden zu absolvieren haben. Obwohl in der BolognaErklärung - und auch in den Konkretisierungen in den nationalen Richtlinien - in keiner Form vorgeschrieben wird, wie viele und welche Prüfungen im Laufe eines Studiums abzulegen sind, ist nicht zu bestreiten, dass es im Vergleich zu den alten Studiengängen zu einer erheblichen Erhöhung der Prüfungs- und damit auch Korrekturlasten gekommen ist. Es gibt Universitäten, in denen Studierende neben den regelmäßig in den Veranstaltungen zu erbringenden Schreib- und Präsentationsleistungen pro Semester mehr als sechs benotete und endnotenrelevante Prüfungen in Form von Klausuren, Hausarbeiten oder Literaturberichten erstellen müssen (vgl. die Diagnose bei Draheim/Reitz 2005, 7).

Diese "Prüfungsinflation« ist quasi automatisch im Zuge der Einführung der Modulstruktur an den Universitäten entstanden. Da sich an den Hochschulen im Stile eines Stille-Post-Effekts die Auffassung durch gesetzt hat, dass jedes Modul mindestens eine Einzelleistung enthalten müsse und prüfungsleistungsfreie Module nicht vorkommen sollen, wird automatisch mit der Zahl der Module in einem Studium gleichzeitig die Zahl der abzulegenden Prüfungen festgelegt. Wenn in einem aus 180 Leistungspunkten bestehenden BachelorStudiengang die Module durchschnittlich aus fünf Leistungspunkten bestehen, bedeutet dies, dass zur Absolvierung von sechs Modulen pro Semester auch verpflichtend sechs Prüfungen in irgendeiner Form abgelegt werden müssen und damit dreißig - in der Regel endnotenrelevante - Prüfungen im Rahmen eines lediglich dreijährigen Studiums. Die Hochschulen - und im Besonderen die Fachbereiche - bestimmen also letztlich mit der Entscheidung über die Modul-
größen automatisch auch über die Zahl der zu absolvierenden Einzelleistungen. 
Mit der damit einhergehenden Vervielfältigung der Prüfungslasten wird dann in vielen Fällen auch die Prüfungsform bestimmt. So mag es aus didaktischen Gründen sinnvoll sein, in einem Studiengang von den Studierenden als zu erbringende Leistung eine Mischung aus mehreren Hausarbeiten, Referaten und mündlichen Prüfungen zu erwarten, aber durch die Vervielfältigung von Prüfungslasten ist dies häufig weder von den Studierenden noch von den mit der Korrektur beauftragten Lehrenden zu leisten. So wird dann häufig für ein Modul nicht die didaktisch sinnvolle Prüfungsform gewählt, sondern die Prüfungsform, die vom bestehenden Lehrpersonal mit einem zu vertretenden Aufwand überhaupt noch zu leisten ist. An einigen Universitäten sollen deshalb Lehrende selbst für Module wie »Geschichte des Nationalsozialismus", "Logik in der Philosophie« und »Soziologische Grundbegriffe« bereits Multiple-Choice-Klausuren entwickelt haben, die dann arbeitssparend nicht mehr durch das Lehrpersonal selbst, sondern durch die Sekretärinnen korrigiert werden können.

Eine weitere ungewollte Nebenfolge des Sudoku-Effekts ist die Einschränkung der Wahlmöglichkeiten für Studierende. Auf den ersten Blick suggerieren Tausende von Modulen an einer Universität erst einmal fantastische Wahlmöglichkeiten für die Studierenden. Aber die faktischen Wahlmöglichkeiten werden nicht vorrangig durch die Wählbarkeit von Modulen produziert, sondern durch die Wählbarkeit von Veranstaltungen innerhalb von Modulen. Je mehr Module eine Universität durch ihre kleingliedrige Modulstruktur anbieten muss, desto weniger Wahlmöglichkeiten bestehen bei gleichen Kapazitäten innerhalb der Module. Wenn durch die Lehrenden verpflichtend eine Vielzahl von Modulen angeboten werden muss, können sie innerhalb der Module häufig kaum noch alternativ verschiedene Seminare, Vorlesungen oder Übungen präsentieren.

Der Grund dafür mag etwas kompliziert erscheinen, aber eigentlich ist es ganz einfach: Vor der Einführung der Bologna-Studiengänge konnten Studierende, jedenfalls in den Geistes- und Sozialwissenschaften, aus einem breiten Spektrum von angebotenen Veranstaltungen frei wählen. Sie mussten beispielsweise in einem Vertiefungsgebiet lediglich von den zwölf in einem Jahr angebotenen Veranstaltungen zur Geschichte des neunzehnten und zwanzigsten Jahrhunderts, zur soziologischen Theorie oder zur Botanik sechs Seminare auswählen. Selbst wenn man zeitliche Überschneidungen von Veranstaltungen mit hineinrechnet, hatten Studierende so viele Wahlmöglichkeiten, dass es für Mathematiker nicht einfach ist, die in die Tausende gehenden Kombinationsmöglichkeiten zu errechnen.

Mit der Einführung der kleingliedrigen Modulstruktur wurden diese in einem Jahr angebotenen zwölf Veranstaltungen jetzt - nur um ein Beispiel zu nennen - auf drei jeweils mit genauen Lernzielen beschriebene Module verteilt. Statt eines großen Containers "Geschichte des neunzehnten und zwanzigsten Jahrhunderts " gibt es jetzt beispielsweise drei Module mit den
Titeln »Geschichte der Industrialisierung», "Geschichte totalitärer Regime« und "Geschichte demokratischer Staaten", die jeweils aus zwei Seminaren bestehen; statt eines umfassenden Vertiefungsgebiets "Soziologische Theorie" werden jetzt drei Module mit jeweils zwei Seminaren angeboten, eines für "Gesellschaftstheorie«, eines für »Organisationstheorie« und eines für »Interaktionstheorie«; das Vertiefungsgebiet »Botanik« wird in die drei Module »Pflanzenmorphologie", "Pflanzenphysiologie" und "Pflanzensystematik" zerlegt. Wenn man jetzt - mit Verweis darauf, dass man die in einem Modul vermittelten Kompetenzen ja nur einmal zu erlangen braucht - verbietet, dass Studierende Module mehrfach belegen und stattdessen in diesem Vertiefungsgebiet das Belegen aller drei Module festschreibt, kann man sehen, wie die Wahlmöglichkeiten der Studierenden sich reduzieren. Weil bei gleichen Lehrkapazitäten pro Modul in einem Jahr nur vier Seminare angeboten werden, können sich wegen der Kollision mit anderen Veranstaltungen die Wahlmöglichkeiten faktisch auf null reduzieren.

Die Aufschlüsselung der Studiengänge in eine Vielzahl von kleingliedrigen, sehr genau definierten Modulen bedeutet für Studierende also die "größtmögliche ungewollte Vernichtung aller Wahlmöglichkeiten« (kurz "GUVAW«). Durch die detaillierte Aufgliederung in manchmal auch noch unterschiedlich große Module kommt es ungewollt zu einer weitgehenden Vorbestimmung des Studienverlaufs, weil Studierende letztlich vielfach die Module, Veranstaltungen und Prüfungen wählen müssen, die irgendwie noch in die durch den Sudoku-Effekt geprägte komplexe Modulstruktur passen.

\section{Der Teufelskreis einer permanenten Hochschulreform}

Die Effekte der Hochschulreform in Form von Komplexitätssteigerung und weitergehend in Form einer zunehmenden Verschulung werden auch in den Hochschulen beobachtet - ohne dass diese Effekte aber als ungewollte Nebenfolge der in Modulen zusammengefassten und in ECTS-Punkten ausgedrückten Veranstaltungen und Prüfungen identifiziert wird. Als Reaktion kommt es deswegen immer wieder zu mehr oder weniger umfassende Reformmaßnahmen an den Hochschulen. Dabei werden dann die Studiengänge einer ganzen Universität zum Beispiel durch die Vorgabe einer einheitlichen Modulgröße neu konzipiert oder neue spezifische Anweisungen zum Beispiel über Anzahl von Prüfungen oder Vorlesungen gegeben, die universitätsweit in allen Studiengängen umzusetzen sind. Eine grundlegend neue Studiengangskonzeption soll dann Probleme, Schwierigkeiten und Holprigkeiten der existierenden Studiengänge ausmerzen.

Manche Hochschulen verfangen sich auf diese Weise in einem Teufelskreis permanenter, aufeinander aufbauender Reformen, und es gibt Bachelor- und Master-Studiengänge, die sich in ihrem jungen Leben bereits in der dritten 
Reformrunde befinden. Das liegt daran, dass ein solches, durch eine Reform angestoßenes neues Regelwerk immer erst einmal eine eigene Attraktivität hat. Im Vergleich zum alltäglichen, häufig zähen und für viele Studierende, Lehrende und Verwaltungsmitarbeiter frustrierenden Zusammenwirken im Rahmen der existierenden Studienstrukturen malt eine Reform erst einmal "schöne Bilder" einer stromlinienförmigen und widerspruchsfreien Studienstruktur. Weil im Vergleich zu der als chaotisch wahrgenommenen Realität die Masterpläne für eine neue Studienstruktur attraktiver, einfacher und einleuchtender wirken, entsteht zunächst die Plausibilität für eine Umsetzung einer neuen Studienstruktur. Schließlich sind die Pläne in »ihren guten Absichten nur schwer zu widerlegen«, weil der »Härtetest ihrer Vorhaben« noch aussteht (Luhmann 2000, 338).

Aber in ihrer Umsetzung verlieren solche Reformen dann immer wieder ihre Attraktivität. Je konkreter ein Masterplan für eine neue Studienstruktur in die Realität umgesetzt wird, desto klarer wird, dass dieses Konzept ähnlich viele Widersprüchlichkeiten birgt wie alle anderen, vorher bekannten Organisationskonzepte auch. Plötzlich wird dann deutlich, dass das als Wundermittel verkaufte Konzept der gleich großen Modulgrößen, die Schwierigkeit mit sich bringt, auch aufwendige Veranstaltungen in das Korsett einzupassen. Auf diese Abnutzung einer Reformkonzeption wird dann wieder mit neuen Reformen reagiert, die selbstverständlich mit dem Versprechen höherer Konsistenz und Handhabbarkeit präsentiert werden. Diese Reform schafft aber wiederum neue Probleme, und die werden dann zum Anlass genommen, die nächste Reform zu starten. Der Effekt der Umsetzung der Bolonga-Vorgaben ist deswegen an einigen Universitäten eine Art »Dauer-Reform " gewesen. Denn Reformen, so Nils Brunsson und Johan P. Olsen (1993, 33f.) produzieren vorrangig immer wieder neue Reformen

\section{Literatur}

Baecker, Dirk (1992): Fehldiagnose "Überkomplexität«. Komplexität ist die Lösung, nicht das Problem. gdi impuls, Nr. 4, 55-62.

BLK (2002): Modularisierung in Hochschulen. Handreichung zur Modularisierung und Einführung von Bachelor- und Masterstudiengängen. Bonn: Bund-Länder-Kommission für Bildungsplanung und Forschungsförderung.

Bologna-Erklärung (1999): Der Europäische Hochschulraum. Bologna: Gemeinsame Erklärung der Europäischen Bildungsminister 19.6.1999.

Brunsson, Nils/Olsen, Johan P. (1993): The Reforming Organization. London/New York: Routledge.

Budapest-Wiener Erklärung (2010): Erklärung von Budapest und Wien zum Europäischen Hochschulraum. Budapest / Wien: Erklärung der Bildungsminister am 12.3.2010.

Draheim, Susanne/Reitz, Tilman (2005): Währungsreform. Die neue Ökonomie der Bildung. Neue Sammlung 45, 3-13
Erhardt, Manfred (2000): Einleitung. S. 3-4 in: Stifterverband für die Deutsche Wissenschaft (2000): Credits an deutschen Hochschulen. Transparenz - Koordination - Kompatibilität. Bonn: Stifterverband für die Deutsche Wissenschaft.

Gaston, Paul L. (2010): The Challenge of Bologna. What United Stats Higher Educaiton Has to Learn From Europe, and Why It Matters That We Learn It. Sterling, VA: Stylus.

Heublein, U. et al. (2007): Internationale Mobilität im Studium. Studienbezogene Aufent halte deutscher Studierender in anderen Ländern. Hannover: HIS:Projektbericht.

Kapfinger, Emanuel/Sablowski, Thomas (2010): Bildung und Wissenschaft im Kapitalismus. S. 249-278 in: Horst, Johanna-Charlotte (Hrsg.), Unbedingte Universitäten. Was passiert? Stellungnahmen zur Lage der Universität. Zürich: Diaphanes.

Kieserling, André (2009): Die Wirklichkeit der Humboldt-Rhetorik oder Was soll aus den Studenten werden? S. 26-37 in: Jürgen Kaube (Hrsg.), Die Illusion der Exzellenz. Lebenslügen der Wissenschaftspolitik. Berlin: Klaus Wagenbach.

KMK (2004): Rahmenvorgaben für die Einführung von Leistungspunktsystemen und die Modularisierung von Studiengängen. o. O.: Beschluss der Kultusministerkonferenz vom 15.9.2000 i. d. F. vom 22.10.2004

Lieb, Wolfgang (2009): Humboldts Begräbnis. Blätter für deutsche und internationale Politik, H. 6, 89-96.

Liessmann, Konrad Paul (2009): Vorwort. S. 7-10 in: Andrea Liesner/Ingrid Lohmann (Hrsg.), Bachelor bolognese. Erfahrungen mit der neuen Studienstruktur. Opladen/Farming. ton Hills: Verlag Barbara Budrich.

Luhmann, Niklas (1972): Rechtssoziologie. Reinbek: Rowohlt.

Luhmann, Niklas (2000): Organisation und Entscheidung. Opladen: Westdt. Verlag.

Luhmann, Niklas (1980): Komplexität. S. 1064-1070 in: Erwin Grochla (Hrsg.), Handwör terbuch der Organisation. Stuttgart: Poeschel.

Maeße, Jens (2010): Die vielen Stimmen des Bologna-Prozesses. Zur diskursiven Logik eines bildungspolitischen Programms. Bielefeld: transcript.

Merton, Robert K. (1936): The Unanticipated Consequences of Purposive Social Action. American Sociological Reveiw 1, 894-904

Nassehi, Armin (2009): Bologna-Reform. Mehr universitären Liberalismus wagen. FAZ 25.11.2009. Oelze, Berthold (2010): Für eine kritische Soziologie des Bologna-Prozesses. Soziologie 39,
179-185.

Pfaller, Robert (2010): Der Kampf gegen die Fortentwicklung der Universität zur repressiven Attrappe. S. 51-54 in: Johanna-Charlotte Horst (Hrsg.), Unbedingte Universitaten. Was passiert? Stellungnahmen zur Lage der Universität. Zürich: Diaphanes. Prager Erklärung (2001): Auf dem Weg zum europäischen Hochschulraum. Prag: Kom-
muniqué des Treffens der europäischen Hochschulministerinnen und Hochschulminister am 19.5.2001.

Roscher, Falk (2000): Das operative Regelwerk von Credit-Systemen. S. 45-56 in: Stefanie Schwarz/Ulrich Teichler (Hrsg.), Credits an deutschen Hochschulen: Kleine Einheiten große Wirkung. Neuwied/Kriftel: Luchterhand.

Schwarz, Stefanie/Teichler, Ulrich (2000): Memorandum zur Einführung eines CreditSystems an den Hochschulen in Deutschland. Rahmenvorschlägung eines Credit der Studien- und Prüfungsorganisation. S 5-10 in: Stifterverband zur Verbesserung Wissenschaft (2000) Credits an deutschen 5 Kompatibilität. Bonn: Stifterverband für diochulen. Transparenz - Koordination -

Konnon Claude Claude Shannon (1951)

Bell System The Claude Shannon (1951): Prediction and Entropy of Printed English. The Bell System Technical Journal 30, 50-64

Steinert, Heinz (2010): Die nächste Universitätsreform ist schon da. Soziologie 39, 310-324.
Stichweh, Rudolf (1994): Akademische Freiheit Profesionalisierung deg

Stichweh, Rudolf (1994): Akademische Freiheit, Professionalisierung der Hochschullehre und Politik. S. 337-362 in: Rudolf Stichweh (Hrsg.), Wissenschaft, Universität, Professionen. Soziologische Analysen. Frankfurt a. M.: Suhrkamp.

Weizsäcker, Ernst von (1970): Baukasten gegen Systemzwänge. München: Piper 
Winter, Martin (2009): Das neue Studieren. Chancen, Risiken, Nebenwirkungen der Studienstrukturreform: Zwischenbilanz zum Bologna-Prozess in Deutschland. Wittenberg: Arbeitsbericht des Instituts für Hochschulforschung.

Wissenschaftsrat (2000): Empfehlungen zur Einführung neuer Studienstrukturen und -abschlüsse (Bakkalaureus/Bachelor - Magister/Master) in Deutschland. Wissenschaftsrat, 21.1.2000.

Prof. Dr. Stefan Kühl

Fakultät für Soziologie, Universität Bielefeld

Postfach 100131 , D-33501 Bielefeld

stefan.kuehl@uni-bielefeld.de 